\title{
Zur Einführung eines wissenschaftlichen Beirats
}

\author{
Jens S. Dangschat $\cdot$ Johann Bacher $\cdot$ Frank Welz
}

Keinerlei Midlifekrise zeigt unser Gemeinschaftsprojekt der Österreichischen Zeitschrift für Soziologie (ÖZS). Ganz im Gegenteil. Auch in ihrem 36. Jahr lassen zwei Faktoren unser wissenschaftliches Flaggschiff im fluktuierenden Feld der sozialwissenschaftlichen Zeitschriften gegenwärtig sehr gut dastehen. Erstens steht eine routinierte wie breit aufgestellte Redaktion dahinter, in welcher die verschiedenen Hochschulstandorte ebenso wie die landesweit vertretenen Forschungsinteressen repräsentiert sind. Zweitens hilft der Umstand, dass unsere Zeitschrift als Mitgliederorgan von allen derzeit 400 Mitgliedern unserer Fachgesellschaft verbindlich bezogen wird.

Auf der einen Seite hat die ÖZS nach Ende ihrer Jugendphase entsprechend dem „,unternehmerischen“ Zeitgeist der 1990er Jahre, aber auch auf Druck von aus dem Größenwachstum des Faches stammenden Anforderungen akademischer und professioneller werden müssen (Reichmann 2011). Seit Jahren gilt für ihre „offenen Hefte“, die zumindest zwei Mal im Jahr erscheinen, strikt das Double-blind-Peer-Review-Verfahren der Auswahl und Verbesserung der eingereichten Beiträge. Die Erfolgsquote einer Publikation bei der ÖZS liegt - nach praktisch obligatorischer Überarbeitung jeder Einreichung - bei ungefähr vierzig Prozent (Niedenzu 2011, S. 6). Auf der anderen Seite bewahrt sich die ÖZS einen genuinen Aspekt „Made in Austria“. Dieser besteht darin, jährlich neben den offenen Heften zwei „Themenhefte“ herauszugeben, zu welchen die Beiträge teils auch aktiv eingeworben werden. Dabei galt in langfristiger Betrachtung selbst für die offenen Hefte noch stets, dass die Mehrzahl der in der ÖZS publizierten Beiträge aus österreichischen Forschungsstätten eingereicht wurden - mit Ausnahme der jüngsten

(C) VS Verlag für Sozialwissenschaften 2012

Dr. Jens S. Dangschat $(\bowtie)$

Fachbereich Soziologie, Technische Universität Wien,

Paniglgasse 16, 1040 Wien, Österreich

E-Mail: jens.dangschat@tuwien.ac.at

Dr. Johann Bacher

Institut für Soziologie, Universität Linz,

Altenberger Str. 69, 4040 Linz, Österreich

E-Mail: johann.bacher@jku.at

Dr. Frank Welz

Institut für Soziologie, Leopold-Franzens-Universität Innsbruck,

Universitätsstr. 15, 6020 Innsbruck, Österreich

E-Mail: frank.welz@uibk.ac.at 
Fünfjahresperiode 2006-2010, in welcher erstmals die Einreichungen aus Deutschland überwogen (Reichmann 2011, S. 18).

Eine wichtige Neuerung der letzten Jahre mit noch offenem Ausgang ist, dass die ÖZS als eine Zeitschrift des Springer VS Verlages jetzt auch via SpringerLink an weltweit derzeit 6175 Institutionen digital verfügbar gemacht wurde. Zum Beispiel darf sich die hier vorangegangene Kölner Zeitschrift für Soziologie und Sozialpsychologie binnen der wenigen Jahre von 2005 bis 2008 einer Vervierfachung ihres Impact-Faktors im Social Science Citation Index (SSCI) erfreuen, die laut Verlagsangaben auf die digitale Verfügbarkeit zurückgeführt werden muss. Zwar liegen die Download-Zahlen an Beiträgen aus der Kölner Zeitschrift mit über 100.000 Downloads pro Jahr weit über den Vergleichszahlen der ÖZS. Aber dennoch erreichen auch letztere Dimensionen, mit welchen die vormalige ausschließliche Regalhaltung der papiernen Zeitschrift sicher nicht konkurrieren kann. So steht zu erwarten, dass der ÖZS-Beitrags-Download 2011 den bisherigen Höchstwert aus dem Jahr 2009 (17.795) übertrifft, nachdem 2010 bei unserer Zeitschrift und benachbarten Zeitschriften ein gewisses Absinken der Zahl auffiel (auf 14.737 Downloads). ${ }^{1}$ Ein Grund für die stark gewachsene Nachfrage nach deutschsprachigen Zeitschriften könnte neben der Verbreitung der digitalen Verfügbarkeit sein, dass es beispielsweise in manchen asiatischen Ländern bereits als Distinktionsmerkmal gelten soll, nicht lediglich auf englischsprachige, sondern auch auf deutschsprachige Diskurse referieren zu können.

Im Zusammenhang dieser Entwicklungen diskutierten wir schon vor zwei Jahren im Rahmen unseres nationalen Kongresses in Graz darüber, wie die ÖZS vielleicht noch geeigneter im Zeitschriftenmarkt platziert werden könne. Die erste Frage, die sich hier stets stellt, ist, ob man sich um eine Aufnahme in den SSCI bemühen sollte. Benachbarte Zeitschriften tun dies aktuell, und gerade im vergangenen Jahr wuchs die Zahl der im SSCI indexierten Soziologie-Zeitschriften um ein Drittel an. In der SSCI-Rubrik Soziologie sind als deutschsprachige Zeitschriften bislang vertreten: Berliner Journal für Soziologie, Kölner Zeitschrift für Soziologie und Sozialpsychologie, Soziale Welt, Zeitschrift für Soziologie. Eine Ausrichtung der Zeitschrift auf dieses Ziel wurde sehr unterschiedlich bewertet. Über andere Ansätze, unsere Zeitschrift noch stärker ins Bewusstsein der deutschsprachigen Soziologie zu heben, gab es dagegen hohen Konsens. Eine Möglichkeit wäre, zu versuchen, die früheren Ausgaben der ÖZS in JSTOR oder, wie die Österreichische Zeitschrift für Politikwissenschaft, im Science Open Access Repository (SSOAR) digital und in freiem Zugang anzubieten. Eine andere Option wäre, den Erfahrungen der Kölner Zeitschrift für Soziologie und Sozialpsychologie folgend den Service-Teil der Zeitschrift mit seinen Besprechungen, Ankündigungen, Hinweisen usw. auszubauen.

Im Kontext dieser Überlegungen einer weiteren Profilierung wurden wir im Vorstand als Herausgeber der ÖZS von der Redaktion gebeten, uns in jedem Falle der Einführung eines wissenschaftlichen Beirates für die ÖZS anzunehmen. Ein dann von Frank Welz - nach gründlichem Informationsaustausch mit Vertretern vergleichbarer Fachzeitschriften - koordinierter Vorschlag von zehn publikationsstarken und erfahrenen Wissenschaftlerinnen und Wissenschaftlern wurde im Vorstand und insbesondere auch in der Zeitschriftenredaktion ausführlich erörtert und sehr positiv aufgenommen. Nach einer in

1 Unser besonderer Dank gilt Andreas Beierwaltes (VS Verlag, Cheflektor Soziologie), der uns die angegebenen Daten zur Verbreitung der ÖZS zur Verfügung stellte. 
der Generalversammlung der ÖGS im Dezember 2010 begonnenen breiteren Diskussion war klar, dass für den Beirat renommierte SozialwissenschaftlerInnen gewonnen werden sollten, die außerhalb Österreichs arbeiten, die deutsche Fachliteratur lesen können und die soweit für die Arbeit und das Profil der ÖZS zu gewinnen waren, dass sie die Redaktion und den Vorstand (als Herausgeber) über einen gewissen Zeitraum konstruktiv-kritisch begleiten. Zusätzlich war es uns wichtig, das Fach in der nötigen Breite durch die BeirätInnen repräsentiert zu sehen und auch zu einer geeigneten regionalen Verteilung zu gelangen - hier waren die guten Kontakte zur European Sociological Association (ESA) sehr hilfreich.

Besonders erfreulich für uns war, dass ausnahmslos alle eingeladenen FachvertreterInnen zusagten. Aufgrund der vertretenen Arbeitsgebiete von der Arbeits- bis zur Wissenschaftssoziologie, von Mikro- wie Makroansätzen, Theorie und Geschichte der Soziologie bis hin zur quantitativen und qualitativen Sozialforschung scheint uns eine komplementär sich ergänzende Diversität der Ansätze und Themen auf eine Weise garantiert, die die verschiedenen, im Rahmen unserer Fachgemeinschaft in Österreich vorhandenen Interessen abzubilden vermag. Zudem handelt es sich in allen Fällen um Soziologinnen und Soziologen, die durch deutschsprachige Publikationen, Forschung, Habilitation, Lehre oder eigenes Studium in Österreich, der Schweiz oder in Deutschland durchaus über einen starken Bezug zur deutschsprachigen Soziologie, teils auch eine persönliche Verankerung verfügen. Aus unserer Sicht sind die gewonnenen Beiratsmitglieder sehr geeignet dafür, das HerausgeberInnengremium sowie die Redaktion der Zeitschrift in den Belangen des laufenden Geschäfts zu beraten, eine Außenbeobachtung, Autorengewinnung, vielleicht auch einmal eigene Beitragseinsendung beizusteuern und zuvörderst die ÖZS nach außen zu repräsentieren. Wir freuen uns, Ihnen die BeirätInnen hier vorstellen zu dürfen (in alphabetischer Reihenfolge):

\begin{tabular}{ll}
\hline Name & Institution \\
\hline Marek Czyzewski & Universität Lodz, Institut für Soziologie \\
Kurt Imhof & $\begin{array}{l}\text { Universität Zürich, Forschungsbereich Öffentlichkeit und } \\
\text { Gesellschaft } \\
\text { Boston University, Department of Sociology }\end{array}$ \\
Stephen Kalberg & University of Chicago, Department of Sociology \\
Karin Knorr Cetina & Universität Bamberg, Institut für Soziologie \\
Richard Münch & Newcastle University, School of Geography, Politics and Sociology \\
William Outhwaite & $\begin{array}{l}\text { Université Paris Descartes, Faculté des Sciences Humaines et } \\
\text { Sociales }\end{array}$ \\
Jan Spurk & $\begin{array}{l}\text { State University of Rio de Janeiro, Institute of Social and Political } \\
\text { Studies }\end{array}$ \\
Frédéric Vandenberghe & University of California, Berkeley, Department of Sociology \\
Loïc Wacquant & University of Aberdeen, School of Social Science \\
Claire Wallace &
\end{tabular}


Alle Beiratsmitglieder haben ihr großes Interesse bekundet und freuen sich auf eine Zusammenarbeit mit der Redaktion und dem Vorstand. Zu einem ersten Treffen mehrerer Mitglieder mit Vertretern des Vorstandes und der Redaktion kam es bereits am Rande des Dreiländerkongresses für Soziologie in Innsbruck im September des Jahres. Der Vorstand bedankt sich im Namen der ÖGS-Mitglieder dafür, dass die Beiratsmitglieder die Aufgabe ehrenamtlich erfüllen.

\section{Literatur}

Niedenzu, Heinz-Jürgen. 2011. Arbeitsbericht der ÖZS-Redaktion über die Offenen Hefte 20022009. Österreichische Zeitschrift für Soziologie 36 (3): 6.

Reichmann, Werner. 2011. 35 Jahre ÖZS - Eine quantitative Bestandsaufnahme. Österreichische Zeitschrift für Soziologie 36 (3): 7-28. 